\title{
Navigating the Phonology-Syntax Interface and Tri-P Mapping
}

\author{
Taylor L. Miller \\ State University of New York at Oswego
}

\section{Introduction}

This paper introduces a new model of the phonology-syntax interface called Phase-Based Prosodic Phonology (or Tri-P Mapping), building on proposals in Miller 2018. This model provides a better account for patterns observed in cases of extreme morpho-syntactic complexity or "polysynthesis." While it is widely acknowledged that phonological processes may be restricted to certain domains, appearing in a particular location or spanning some - but not all - junctures within (morpho-)syntactic structure, debate centers on how to derive phonological domains. There are three main models in the current literature: Relational Mapping (e.g. Nespor \& Vogel, 1986; Vogel, 2019), Syntax-Driven Mapping (e.g. Selkirk, 2011; Elfner, 2012; Guekguezian, 2017), and the Syntactic Spell-Out Approach (e.g. Pak, 2008; Samuels, 2011; Surkalović, 2011, 2013; Michaels, 2013; McPherson, 2014; McPherson \& Heath, 2016; Ahn, 2015). ${ }^{1}$

Comparisons between specific approaches have been made (see Selkirk 2011 and Vogel 2019), but the only side-by-side test of all three approaches using the same data is found in Miller 2018. As part of that study, I argue extreme morpho-syntactic complexity or "polysynthesis" is the critical and missing test case for any model of the phonology-syntax interface. Therefore, I turn to two such languages as test cases: Kiowa (Tanoan) and Saulteaux Ojibwe (Algonquian). Comparing each model's predictions to those phonological domains observed in the languages, no current model is entirely successfully. Thus, a new model is necessary.

The remainder of this paper is structured as follows. In Section 2, I provide a brief background of each of the main interface models. Section 3 justifies turning to traditionally termed "polysynthetic" languages as the crucial test case for interface models, notably missing from previous interface analyses. In fact, the foundational assumptions of each model yield different predictions for phonological domains in Kiowa and Saulteaux Ojibwe. In 3.2-3.3, I present the results of the two case studies, demonstrating the need for a new interface model. I introduce Tri-P Mapping in Section 4. I begin by detailing the basic assumptions of the model (4.1), define the relevant prosodic domains (4.2), and revisit the Kiowa and Saulteaux data in 4.3. In Section 5 I discuss the success of Tri-P Mapping and remaining questions regarding the role of an intermediate constituent between the Phonological Word and Phonological Phrase.

\section{Delimiting Phonological Domains}

Each of the three main interface models have rich histories in the literature, often including multiple iterations and proposals. For the sake of space, this section includes only a brief introduction to the core

\footnotetext{
* The data in this paper comes from fieldwork on Kiowa in Carnegie, Oklahoma and Saulteaux Ojibwe in Winnipeg, Manitoba. The trips were funded through the Jacobs Research Fund. I am honored to have met and learned from my teachers in both communities. Thanks also to the audience at AMP 2019 and to countless advisors and colleagues throughout the past several years. In particular, though, thanks to Irene Vogel, Jeffrey Heinz, Benjamin Bruening, and Laura McPherson for advising my dissertation work on this subject. All errors are my own.

1 A fourth movement in the literature has been called the Phenomenon-Based Approach by Vogel 2019. Stemming primarily from the AUTOTYP database (Bickel \& Nichols, 2001), work using this method includes Voll 2006; Hildebrandt 2007; Bickel et al. 2009; Schiering et al. 2007, 2010. Miller 2018 and Vogel 2019 have shown that the approach is merely descriptive and untestable. Therefore, it has been excluded from the present paper. Interested readers are directed to Miller and Vogel's discussions for further information.
} 
of each model and focuses primarily on the most recent work. ${ }^{2}$ The models' differing assumptions are also examined, as they yield different predictions for the size and shape of phonological domains in a given language. As shown in Table 1, there are four areas of interest: reference strategy, syntactic objects referenced, the separation of Morphology and Syntax, and if recursion is allowed.

\begin{tabular}{r|c|c|c|c} 
Model & Reference Strategy & Syntactic Objects & Separate Morpho. & Recursion \\
\hline Relational Mapping & Indirect & Classic & Yes & No \\
Syntax-Driven Mapping & Indirect & Classic & No & Yes \\
Syntactic Spell-Out & Direct & Classic & No & Yes
\end{tabular}

Table 1: Comparing Current Models

There are two reference strategies (or the manner in which phonology references morpho-syntactic structure) adopted in the literature. Direct Reference models read phonological domains directly from syntactic structure, while Indirect Reference models reference morpho-syntactic structure when deriving independent prosodic constituents that may result in non-isomorphism between phonological and morphosyntactic structure (see Shwayder 2015 for a larger discussion on the distinction). In terms of syntactic structure, some models reference classic syntactic objects like heads, phrases, and clauses $\left(\mathrm{X}^{0}, \mathrm{XP}, \mathrm{CP}\right)^{3}$ while others reference phases or spell-out domains. As discussed in Section 2.3 below, precise definitions of phases and their behaviors also differ across models. If a model assumes Morphology is a separate module from Syntax, phonology is permitted to reference morphological structure (e.g. roots, affixes, clitics).

Finally, regardless of what and how phonology references, models differ in whether or not phonological domains may exhibit recursion. It has generally been argued that the presence of recursion is one of the main differences between phonology and syntax (Jackendoff \& Pinker, 2005; Pinker \& Jackendoff, 2005; Neeleman \& van de Koot, 2006; Van der Hulst, 2010; Heinz \& Idsardi, 2011), though the idea of allowing some kind of recursion in phonology (especially at higher prosodic levels) is not new (e.g. Ladd, 1986). As discussed below, some interface models allow pervasive recursion throughout phonological domains at all levels, while other restrict it or ban it altogether. The nature of that recursion, and whether it behaves the same as in syntax, is a point of debate.

2.1 Relational Mapping Relational Mapping, first formalized as Prosodic Phonology (Selkirk, 1980, 1981a,b; Nespor \& Vogel, 1986), is an indirect reference model that builds independent prosodic constituents that layer and build according to size and character to form the Prosodic Hierarchy (Nespor \& Vogel, 1986). Vogel 2019 lists the following universal prosodic constituents: Syllable $(\sigma)$, Foot $(\Sigma)$, Prosodic Word $(\omega)$, Composite Group $(\kappa)$, Phonological Phrase $(\varphi)$, and Intonational Phrase ( $\iota)$. Prosodic Phonology originally assumed the Strict Layer Hypothesis (SLH), a ban on level-skipping and recursion in the prosodic hierarchy. Later work recognizes a need for level-skipping (e.g. Truckenbrodt, 1995, 1999; Selkirk, 1995, 2000; Vigário, 2010; Vogel, 2008, 2009a,b, 2019), however, so the SLH is either noticeably weakened or absent in current work. Vogel's Composite Prosodic Model, the most recent work in Relational Mapping, takes a mixed approach banning recursion only below the Phonological Phrase (Vogel, 2019).

The Composite Prosodic Model divides the Prosodic Hierarchy into three parts: non-interfacing constituents $(\sigma, \Sigma)$, those at the Morphology Interface $(\omega, \kappa)$, and those at the Syntax Interface $(\varphi, \iota)$. The main difference between the two interface groupings is the direction of the mapping procedure. At the Morphology Interface, mapping is a bottom-up procedure meaning that prosodic structure is built up referencing morphological information and including phonologically cohesive elements. For example, the Phonological Word is required to minimally consist of a morphological root. Other material may also be included (i.e. cohering affixes). Any stray elements (i.e. adjoining affixes, clitics, function words) join the $\omega$ at the next level: the Composite Group $(\kappa)$. In addition, $\kappa$ forms the domain for compounds.

In contrast, prosodic constituents are mapped top-down at the Syntax Interface primarily referencing syntactic structure and thus demonstrating more markedly syntactic properties like recursion. In fact, Vogel argues it is only at these levels true recursion is observed (i.e. the exact same phonological processes

\footnotetext{
2 For a more detailed history of each model and illustrative examples, interested readers are directed to those works cited and to Miller 2018.

3 Note that $\mathrm{X}^{0}$ here refers to a terminal node in the syntax. Specifically, that $\mathrm{X}$ must be a lexical head like N, V, or A.
} 
applying repeatedly in each nested structure). The Phonological Phrase consists of the lexical head (N, $\mathrm{V}, \mathrm{A}$ ) of a syntactic phrase. The head's complement is optionally included, depending on whether or not it patterns phonologically with the head. The Intonational Phrase maps from CPs (root sentences, parenthetical expressions, nonrestive relative clauses, tag questions, vocatives, expletives, and certain moved elements).

As the absence of the SLH significantly opens up the prosodic hierarchy and its properties, the CPM aims to restrict the model's power using a combination of three properties: Constituent Sequencing, Proper Headedness, and the Principle of Minimal Distance. Constituent Sequencing, the restriction that a constituent cannot dominate a constituent of its same type, is found at the Morphology Interface thus ruling out recursion at these levels. The remaining two properties are true of the whole hierarchy. Namely, Proper Headedness requires that a constituent dominate at least one constituent of the immediately lower level, and the Principle of Minimal Distance requires that phonological material be parsed into prosodic structure as soon as possible. In both cases, these properties greatly restrict level skipping.

It is Relational Mapping's use of the intermediate constituent $\kappa$ (originally proposed as the Clitic Group in Nespor \& Vogel 1986) that is met with the most criticism (Vogel, 2009a). Frequently, it is seen as unnecessary because it often overlaps with $\omega$ s. Other times, it is omitted entirely in favor of recursive $\omega$ structures - and an overall simpler Prosodic Hierarchy - to account for the same phenomena. Vogel argues the first issue is not a problem. That $\omega$ and $\kappa$ often coincide does not negate instances where they necessarily do not. As for the second issue, Vogel argues against the use of recursive $\omega \mathrm{s}$. At its core, recursion requires that an entity of a certain type be contained within another entity of that type (e.g. XP within XP). The nested entities retain the same category label because they share all characteristics. In fact, the phenomena often associated with recursive $\omega$ structures (e.g. clitic-specific processes or compound phonology) are notably different from the lower $\omega$. If it were a case of genuine recursion, the same phonological processes would be observed at both levels. As this is not the case, Vogel argues this is confirmation for an intermediate constituent.

2.2 Syntax-Driven Mapping Syntax-Driven Mapping is also an Indirect Reference model, but phonology references only syntactic structures. Morphology is subsumed under a "syntax all the way down" approach. When building the Prosodic Hierarchy, phonology references classic syntactic constituents $\left(\mathrm{X}^{0}\right.$, $\mathrm{XP}, \mathrm{CP}$ ), and $\kappa$ is omitted from the hierarchy. The most recent work using this model is Match Theory (Selkirk, 2011), which proposes a set of faithfulness constraints requiring prosodic and syntactic constituents match or coincide: Match Word $\left(\omega \cong X^{0}\right)$, Match Phrase $(\varphi \cong X P)$, and Match Clause $(\iota \cong \mathrm{CP})$. A series of markedness constraints pertaining to the phonological characteristics of the prosodic domains, if highly ranked, allow violations and yield non-isomorphic structures. For example, Selkirk uses Elordieta's (2006; 2007) analysis of Lekeito Basque to show the need for a constraint against non-binary $\varphi s$ at the beginning of the $\iota$ domain.

Match Theory (Selkirk, 2011) goes beyond previous Syntax-Driven Mapping approaches (e.g. Alignment Theory, Wrap Theory) and assumes recursion to not only be possible but prevalent based on the syntactic structure. Genuine recursion is exhibited at the $\varphi$ and $\iota$ levels (see Selkirk's Match Theory analysis of Xitsonga) because the prosodic constituents in question retain the same phonological characteristics regardless of their position in the structure (e.g. the same phonological processes apply at each successive level). As argued in Vogel 2009a, 2019, this is not possible at the $\omega$ level. As proposed, Match Theory incorrectly predicts recursive $\omega$ structures.

While a syntax-only model is theoretically preferred as it offers a simpler account for grammar, referencing $\mathrm{X}^{0}$ to map $\omega$ leads to issues for Match Theory. As to be discussed below, this has major implications for incorporating languages like Kiowa, but stray elements like Level 2 affixes also cause problems. Because Level 2 affixes form one grammatical word or $\mathrm{X}^{0}$ with the rest of the word, they are automatically parsed into one $\omega$. Vogel 2019 shows that this causes two generalizations to be lost entirely: 1) Level 2 affixes do not pattern with the rest of the grammatical word and 2) Level 2 affixes pattern with other stray elements like clitics and function words. In addition, Vogel argues Match constraints incorrectly predict certain elements should pattern differently due to differences in syntactic structure when they, in fact, do not. For example, English demonstrates a single Voicing Assimilation rule for its various $-s$ morphemes (plural, third person singular, possessive, copula, and auxiliary). Because each of these morphemes attach at different levels of syntactic structure, Match incorrectly predicts them to act differently from one another or, conversely - requires separate phonological rules of Voicing Assimilation for each morpheme. 
2.3 Syntactic Spell-Out Phonology only references syntactic structure in a Syntactic Spell-Out Approach, as well, but phonological application is restricted to phases and not classic syntactic constituents. Of the three models, the Syntactic Spell-Out Approach is the newest and therefore less standardized. While all relevant work implements Phase Theory (Uriagereka; Chomsky, 2000, 2001, 2008; Citko, 2014; Collins $\&$ Stabler, 2016), particular analyses differ in terms of which syntactic heads trigger Spell-Out (what is a phase head), what phonology may access after Spell-Out (the Phase Impenetrability Condition), and how phonology references the structure itself (Direct or Indirect Reference). For this paper, I assume the most recent and widely adopted stances, though the variation will certainly be addressed in Sections 4 and 5.

First, phase heads of two types are assumed: clause-level (C and v/vOICE) and morpheme-level (categorizing heads $n, v, a$ ). Phase heads are functional heads which trigger transfer or Spell-Out to syntax's interfaces - semantics/pragmatics (LF) or phonetics/phonology (PF). Spell-Out Domains consist of the complement of head phase head, and Transfer is triggered whenever a new phase head is introduced in the derivation. ${ }^{4}$

In Phase Theory, the Spell-Out Domain is subject to the Phase Impenetrability Condition (henceforth PIC) (Chomsky, 2001), which disallows higher phases access to material which has already been spelled out. Thus, a phase head only has access to the elements in its phase (and the following c-commanding phase head if one exists). Consider, for example, the following structure found in Samuels 2011:75-76. Assume $\alpha, \beta, \gamma$, and $\delta$ are phase heads. Since syntax trees build from the bottom up, $\beta$ and $\alpha$ merge first and thus are accessible to each other in terms of phonology and semantics. $\beta$ projects upward, and the phrase $\beta \mathrm{P}$ is formed. Next, $\gamma$ merges with $\beta$ P. Since a new phase head has been introduced into the tree, $\beta$ 's complement $(\alpha)$ is transferred to PF. Due to the PIC, $\alpha$ is no longer accessible once it is transferred. Thus, The newly introduced $\gamma$ has access to $\beta$ but not to $\alpha$. This process iterates up the tree as seen in (1).

Merge $(\beta, \alpha): \alpha$ is accessible to $\beta$

Merge $(\gamma, \beta \mathrm{P}): \beta$ is accessible to $\gamma . \alpha$ transferred

Merge $(\delta, \gamma \mathrm{P}): \gamma$ is accessible to $\delta . \beta \mathrm{P}$ is transferred.

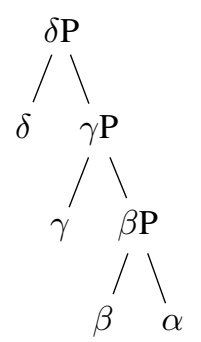

Though originally proposed only for syntactic operations, some work extended the PIC (in various forms) to phonological operations (Wagner, 2005; Samuels, 2009, 2010, 2011). For example, Samuels (2009; 2010; 2011 proposes different versions of the PIC for lexical and post-lexical rules. More recently, researchers have argued against using the PIC in phonology entirely (e.g. Šurkalović, 2011, 2013; Michaels, 2013; McPherson, 2014; McPherson \& Heath, 2016; Ahn, 2015), some proposing to use Phase-Phase Faithfulness Constraints to account for what was originally claimed to be instances of the PIC in Phonology (e.g. McPherson \& Heath, 2016) This paper adopts this recent stance and assumes the PIC does not play a role in phonology.

Finally, this paper assumes a Direct Reference approach in line with work like Pak (2008); Sato (2009, 2012); Samuels (2009, 2010, 2011) and (in a somewhat modified fashion) Samuels (2011). This factor, however, seems to be most varied in the literature. A roughly equal number of researchers have adopted an Indirect Reference strategy and use phases to map to prosodic constituents in the Prosodic Hierarchy (e.g. Cheng \& Downing, 2007; Kratzer \& Selkirk, 2007; Ishihara, 2007; Dobashi, 2003, 2004a,b; Compton \& Pittman, 2007; Piggott \& Newell, 2006; Ahn, 2015). ${ }^{5}$ As a Direct Reference approach is most faithful to Phase Theory, it is assumed here, but the debate is revisited in Sections 4 and 5.

\section{The Missing (and Crucial) Test}

Comparisons between interface models, and analyses within those models, have been made. For example, Selkirk 2011 provides an overview of previous Syntax-Driven approaches (Alignment Theory and

4 See Samuels 2011 for a comprehensive discussion of the debate as to what and what is not a phase head.

5 Šurkalović (2011)'s cumulative Spell-Out has the same effect of an independent prosodic structure, but it is still derived directly from the order of syntactic operations and Spell-Out instead of an independent constituent structure. 
Wrap Theory) and shows that Match Theory can account for the same phenomena. Similarly, Vogel 2019 includes a broad discussion of various Syntax-Driven approaches, as well as Phenomenon-Based analyses (see footnote 2). While important discussions, neither paper is sufficient in order to decide in favor of any particular model. First, Syntactic-Spell Out approaches are not included in either comparison. Second, the comparisons are not a side-by-side evaluation using the same data. In both cases, specific analyses' case studies are merely re-analyzed.

In addition, and perhaps most importantly, no interface proposal has discussed what merits a crucial test for a model of the phonology-syntax interface. Instead, success is claimed for any analysis that provides an account for hand-picked illustrative case studies and which may re-account for other analyses' case studies. In Miller 2018, I argue against this circular reasoning and propose that the crucial test for the phonologysyntax interface is a language with extreme morpho-syntactic complexity. Blurring the lines between the word, the phrase, and the clause pushes a model to its limit. In other words, the crucial - and notably missing - test is what is traditionally described as "polysynthesis." Miller 2018 consists of such a side-by-side test of all three models using languages which demonstrate extreme morpho-syntactic complexity: Kiowa and Saulteaux Ojibwe.

3.1 Current Models' Predictions As expected, the three interface models primarily diverge in their predictions for the most complex word classes in both languages: the verbs. In both languages, the verb complex can form an independent clause through inflection, agreement, and the incorporation of multiple stems. Given the types of objects referenced (classic syntax, phases, and morphological information) outlined in the previous section, the models differ in whether or not they predict verb-internal phonological domains. In addition, reference strategy and the status of recursion in the model also yields differences in predictions for compounds. ${ }^{6}$

First, consider the basic structure of the Kiowa and Ojibwe verbs. As seen in (2), the Kiowa verb complex includes a pronominal prefix (Ppfx), incorporated adverb (Adv), incorporated noun $(\mathrm{N})$, incorporated verb (V), verb stem (STEM), inflectional or modal suffix(es) (I/M), and a syntactic suffix (Synt). Only three elements are obligatory: the pronominal prefix, stem, and an inflectional suffix. Therefore, a verb complex in Kiowa may be very short as in (3) or extremely long as in (4).

(2) Kiowa Verb Complex (Watkins 1984:147)

Ppf $-(A d v)-(N)-(V)-S T E M-I / M ~-~(S y n t)$

$\begin{array}{llll}\text { hón } & \varnothing- & t^{h} p & -\varnothing \\ & \text { Ppfx- } & \text { STEM } & -\mathrm{I} / \mathrm{M} \\ \text { NEG } & {[3 \mathrm{sg}]-} & \text { go.out } & -\mathrm{PF}\end{array}$

'He didn't go out.'

$\begin{array}{lllllll}\text { àn } & \grave{a}- & \text { bô:- } & \text { pòlà:jì- } & \grave{e}:- & \text { bà: } & \text {-mà } \\ & \text { Ppfx- } & \text { Adv- } & \text { N- } & \text { V- } & \text { STEM } & \text {-I/M } \\ \text { HAB } & {[1 \mathrm{sg}]-} & \text { always- } & \text { rabbit- } & \text { hunt- } & \text { go } & \text {-IPFV }\end{array}$

'I'm always going rabbit hunting.'

Miller 2018 provides the following general template for the Ojibwe verb, which varies slightly due to morphological classes based on transitivity and gender. The first element is a pronominal, used for persons other than third person. Originally argued to be a prefix, some argue it is a clitic as shown below (among others Oxford, 2013). This is optionally followed by tense markers and preverbal modifiers. Inflectional endings follow three classes of verb conjugations (Independent, Imperative, Conjunct). There is no limit to the number of preverbs allowed within the complex, though there are rarely instances of more than five (Valentine, 2001; Slavin, 2005). Consider (6), which comes from a longer sentence translating to 'Our darn little teenage turtles, probably never tried to pretend to be smart and wise.' (adapted from Logan 2001:51).

Ojibwe Verb Complex

Pronominal $=$ Tense - Modifier(s) - STEM - Inflection $=$ Obj. Number

6 Unless otherwise cited, all data come from my fieldwork in summer and fall of 2016. 


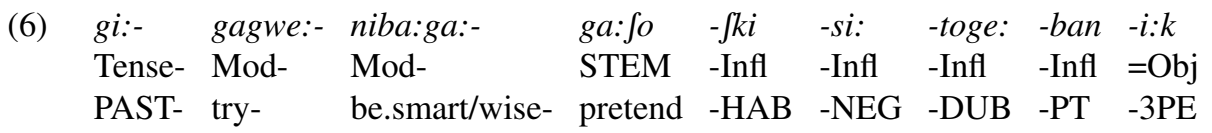

Relational Mapping and Syntactic Spell-Out predict verb-internal domains, while Syntax-Driven Mapping does not. This is primarily due to the fact that Syntax-Driven Mapping references classic syntactic constituents. For both Kiowa and Saulteaux, the verb complex forms a single $\mathrm{X}^{0}$ at the surface regardless of the internal complexity. By referencing only $\mathrm{X}^{0}$ in the definition of Match-Word, the full verb complex is predicted to consist of a single $\omega$ domain. In fact, Match Theory does not have any machinery which accounts for the smaller domains within the verb complex.

Finally, Relational Mapping alone predicts that compounds may exhibit unique phonological patterns. Syntax-Driven Mapping and Syntactic Spell-Out predict compounds to form recursive $\omega$ structures. Therefore, it is necessary to differentiate levels of $\omega$ structures and allow for different phonological characteristics at subsequent levels. This is, by definition, proposing a distinctive prosodic constituent $\kappa$.

3.2 Kiowa Results After analysis of eleven phonological processes in Kiowa, Miller 2018 finds phonological domains of three sizes in the clause (7) and domains of two sizes in compounds (8). Crucially, verb-internal domains are present, and there is evidence of compound-specific phenomena (Tone Raising). Relational Mapping is the only model that successfully predicts each domain.

(7) Phonological Domains in the Kiowa Clause

Domain 1: [Subject] [Object] [Ppfx]-[Adv]-[N]-[V]-[STEM-I/M] -Synt

Domain 2: [Subject] [Object] [Ppfx-Adv-N-V-STEM-I/M-Synt]

Domain 3: [Subject Object Ppfx-Adv-N-V-STEM-I/M-Synt]

(8) Phonological Domains in Kiowa Compounds

Domain 1: $\left[\operatorname{Root}_{1}\right]+\left[\operatorname{Root}_{2}\right]$

Domain 2: $\left[\operatorname{Root}_{1}+\operatorname{Root}_{2}\right]$

Syllabification is restricted to the smaller, verb-internal domains (Clause Domain 1 above). Consider, for example, Cluster Devoicing, which devoices stops following a voiceless obstruent. All morpheme-internal clusters agree in voicing, so the process's application may only be tested at morpheme boundaries. As seen below, Cluster Devoicing only applies across noun-suffix boundaries and the boundary between the STEM and I/M suffix(es) in the verb. The process is blocked at all other morpheme boundaries.

As seen in (9), the perfective suffix /-gjá/ surfaces as [-kjá] following voiceless [t] at the end of the verb root 'write' spanning the STEM-I/M boundary.
$\begin{array}{lll}\text { gját- } & \text { gút } & -k j a ́ \\ \text { [1sg/agt:pl/obj]- } & \text { write } & -\mathrm{PF}\end{array}$
'I wrote it/It was written.'

The process is blocked, however, at every other morpheme boundary within the verb complex. Voiceless stops (resulting from another devoicing process targeting syllable-final obstruents) do not trigger Cluster Devoicing across the prefix's boundary in (10). Likewise the incorporated adverb's final [t] does not trigger the process, and STEM-initial /b/ surfaces unchanged in (11).

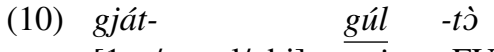

[1sg/agt:pl/obj]- write -FUT

'I will write.'

(11) à- kj̀ét- $\underline{b a ̀ a}$

[1sg]- scared- go.PF

'I fearfully went.'

Cluster Devoicing also does not apply across the boundary between the verb stem and syntactic suffixes. The nominalizing /-gò/ surfaces with [g] instead of a devoiced $[\mathrm{k}]$ after [t] in (12). 


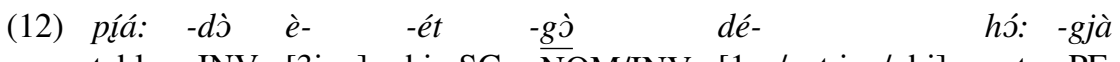
table -INV [3inv]- big.SG -NOM/INV [1sg/agt:inv/obj]- get -PF

'I bought a big table/table that is big. (Watkins 1984:240)'

3.3 Saulteaux Results After analysis of nine phonological processes in Saulteaux, Miller 2018 finds phonological domains of three sizes in the Saulteaux clause (13) and only one in compounds (14). Crucially, one verb-internal domain is observed (Clause Domain 1 below). It involves a phonological boundary between inflectional suffixes, however, and is therefore unpredicted for all three models. No compound-specific phenomena was observed, so the models do not differ. No model achieves overall success for Saulteaux.

(13) Phonological Domains in the Ojibwe Clause

Domain 1: [Pronom=Tense-Mod(s)-Stem-Infl 1 -Infl ${ }_{2}=$ Obj.No. Obj. Subj.

Domain 2: [Pronom=Tense-Mod(s)-Stem-Infl ${ }_{1}-\mathrm{Infl}_{2}=\mathrm{Obj}$.No.] [Obj.] [Subj.]

Domain 3: [Pronom=Tense-Mod(s)-Stem-Infl $1-\mathrm{Infl}_{2}=$ Obj.No. Obj. Subj.

(14) Phonological Domain in Ojibwe Compounds

Domain: $\left[\operatorname{Root}_{1}\right]+\left[\operatorname{Root}_{2}\right]$

It is the interaction between Final Obstruent Devoicing and Final Short Vowel Deletion that indicates a boundary between inflectional suffixes suffixes (abbreviated as $\mathrm{Infl}_{1}$ and $\mathrm{Infl}_{2}$ above). Consider the following data involving the same verb stem /bagizo/ 'swim.' In (15), it is affixed with the third person suffix /-w/. Glide deletion requires final glides to delete, the $/ \mathrm{w} /$ is deleted, and the full stem surfaces.

(15) bagezo /bagizo-w/ swim.3PE

'He is swimming.'

The second person is inflected using a prefix /gi-/, rather than a suffix. In (16)/bagizo/ is complex-final, and Final Short Vowel Deletion and Final Obstruent Devoicing apply.

(16) gi- bages

2PE- swim

'You are swimming.'

As seen in (17), the verb stem again undergoes Final Short Vowel Deletion and Final Obstruent Devoicing even though the environment is not verb complex-final. The application indicates a phonological boundary before the Mode suffix.

(17) ni- wi:- bages -na:ban

1PE- FUT- $\overline{\text { swim }}$-PT

'I was going to swim.'

3.4 Preliminary Discussion Though no model is successful for Saulteaux, future research is necessary. Miller 2018 notes that the data above is the only evidence for the mid-suffixes boundary. All other phenomena apply span the grammatical word and clause, which do not cause issues for any of the three models. It is also unexpected that no processes indicated boundaries between preverbal modifiers, which are independent stems. In addition, Ojibwe stems are traditionally considered not to be monomorphemic and are said to consist of up to three meaningful elements. Their internal structure was not considered in the original analysis, and this may also contribute to the overall understanding of the interface and Saulteaux. Leaving these issues to the side, let us focus on the Kiowa results.

Relational Mapping's success in Kiowa is noteworthy, as it is the only approach which assumes a) an intermediate constituent between $\omega$ and $\varphi$ and b) a strict separation of Morphology and Syntax. Both assumptions are worth examining in detail. First, consider the intermediate constituent $\kappa$. As defined, the full verb complex and compounds are both predicted to form $\kappa$ constituents. All $\kappa \mathrm{s}$ in the language should demonstrate the same characteristic phonological processes, but this is not the case, taking away from 
the model's overall success. The compound-specific process Tone Raising does not apply within the verb complex (18-20). In fact, there is no positive evidence for any characteristic process of the verb complex's $\kappa$ domain.

(18) $\mathrm{k}^{h}{ }^{1}:$ só + dę̀: $\rightarrow \mathrm{k}^{h}$ í:sódę: 'afternoon nap (afternoon + sleep)'

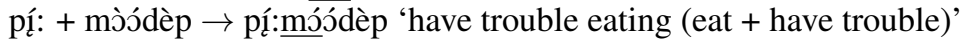

(19) dè-

$$
k^{h} \text { í:só- dè: } \quad-m a ̀
$$

[1sg/agt:3pl/obj]- afternoon- $\overline{\text { sleep }}$-IPFV

'I'm taking an afternoon nap.'

(20) $j a ́ a-$

pị:- $\quad$ mòódèp

[(2,3sg/agt):1sg/pat:pl/obj]- food- have.trouble.PF

'I had trouble eating.'

This asymmetry raises the same question about the definition and predictive power raised for recursive $\omega s$. If two domains don't demonstrate the same phonological characteristics, why are they both called $\kappa \mathrm{s}$ ? Vogel (2009a) anticipates this kind of question for $\kappa$ in Italian. When accounting for the Clitic Vowel Change (CVC) and Clitic Affrication Rule (CAR), she argues the rules apply with regard to the $\kappa$ domain but reference grammatical information within the domain itself. In Italian, she turns to information like the semantic status of the clitics in question. Assuming the same kind of approach in Kiowa, roots forming part of a compound would need to be marked within the $\kappa$ in order to trigger Tone Raising.

Vogel (2009a) argues specifying elements within the domain is preferable to adding more constituents to the Prosodic Hierarchy or introducing recursion, but it is not clear that this is in fact theoretically more desirable. It is true that further specifying components of the domain does not require complicating the possible phonological grammar (i.e. retaining Selkirk 1980's three types of phonological rules: domain span, domain juncture, and domain limit), but referencing additional information makes the concept of the Prosodic Hierarchy more complex. If $\kappa$ domains differ with regard to their individual components, it is not clear there is a benefit of treating them as the same domain. Further, if $\kappa$ must reference domain-internal grammatical information, this must also be true of other prosodic constituents.

Finally, the status of Morphology as a separate module faces theoretical concerns. There is pressure for a linguistic theory to be maximally economic or simple. Thus, a model is preferred if it captures the observed data with the fewest grammatical modules possible. This is to avoid too much power in a model, which may lead to overgeneration and predict structures which are in fact not possible cross-linguistically. While Relational Mapping does not appear to be suffering from overgeneration in the case of Kiowa, a syntaxonly approach which achieves the same level of success is still preferred. I present such an approach in the following section.

\section{Tri-P Mapping: A New Theoretical Proposal}

Tri-P Mapping (Phase-based Prosodic Phonology) is a new model of the phonology-syntax interface, which addresses the successes and failures of the main models tested in Miller 2018. Though open questions remain, a Tri-P analysis achieves the same level of success at Relational Mapping for Kiowa without assuming a morphological module. In addition, it aims to directly address the issues surrounding an intermediate constituent like $\kappa$. In the following subsections, I outline the beginning assumptions of the model (4.1, the definitions of the domains including open questions regarding a Constituent (4.2), and show that Tri-P Mapping achieves the same level of success for Kiowa as Relational Mapping (4.3).

4.1 Beginning Assumptions Tri-P Mapping is a syntax-only Indirect Reference model, which references morpheme-level and clause-level phases to map prosodic constituents. Recursion is banned below $\varphi$, as in Vogel's Composite Prosodic Model. Thus, at least one intermediate constituent is required. This necessitates a new type of reference strategy, as such a domain does not correspond to either type of phase. This will be discussed in detail below.

In terms of Phase Theory, it is assumed that the phase head is included in the Spell-Out Domain (as in Sande \& Jenks 2017; c.f. Bošković 2016). In addition, Phonology may reference any spelled-out phase to 
map to prosodic structure, but phonology itself does not apply cyclically. This allows for domains of smaller sizes, as opposed to work like Cheng \& Downing 2016 which assumes phonology applies after all Spell-Out operations.

4.2 Defining the Domains As in other Indirect Reference Spell-Out accounts (Cheng \& Downing, 2007; Kratzer \& Selkirk, 2007; Ishihara, 2007; Dobashi, 2003, 2004a,b; Compton \& Pittman, 2007; Piggott \& Newell, 2006; Ahn, 2015), morpheme level phases map to $\omega$ and clause level phases little $v$ /VOICE map to $\varphi$. C's phase maps to $\iota$. Phonologically motivated restructuring may then occur including or excluding various elements within the tree. Any constraints on restructuring are left to future research and a larger crosslinguistic analysis, however. In order to account for intermediate structure between $\omega$ and $\varphi$, but continue to avoid referencing morphological information, mapping must be able to reference existing prosodic structure. It is still an open question, though, as to which constituent or constituents are needed.

As discussed earlier, an intermediate constituent like $\kappa$ runs into definitional issues in differentiating between the types of domains and phenomena necessary (i.e. clitic-specific phenomena, function words and other excluded elements, compounds, and domains of different sizes). Half these issues may be considered morphologically-conditioned phonology, though, and are resolved if Tri-P Mapping is combined with another theoretical proposal: CoPhonologies by Phase (Sande, 2017; Sande \& Jenks, 2017). Clitics and function words, for example, would be excluded from Tri-P Mapping entirely, each having its own prosodic specification and constraint rankings in the numeration. The specific phonological patterns are enforced automatically via competition. Any proposal then needs only to account for a domain of an intermediate size accounting for any excluded elements, call it $\chi$, and compounds. Whether or not compounds form an additional prosodic constituent, or a detailed and precise definition of $\chi$, is left to future research.

4.3 Revisiting Kiowa Turning back to Kiowa, and pending a precise account for intermediate structures in the Prosodic Hierarchy, Tri-P Mapping achieves the same level of success as Relational Mapping. Morpheme-level phases predict each incorporated element and the verb stem will form $\omega$ s. Suffixes need to be included in the verb stem's $\omega$ through restructuring. At the clause-level, subject and object NPs are correctly predicted to form $\varphi$ domains due to the phase head $\mathrm{D}$. The object NP and verb complex will initially map to a single $\varphi$ due to VOICE. Restructuring, however, will allow the verb to form its own $\varphi$ separate from the object. The subject and object NPs will each form their own $\varphi$. Finally, the full CP maps to $\iota$.

(21) $\omega$ Domains in the Kiowa Verb

Ppfx $-[\mathrm{Adv}]_{\omega}-[\mathrm{N}]_{\omega}-[\mathrm{V}]_{\omega}-[\mathrm{STEM}-\mathrm{I} / \mathrm{M}-\mathrm{Synt}]_{\omega}$

(22) $\varphi$ and $\iota$ Domains in the Kiowa Clause

$\left[[\text { Subject }]_{\varphi}[\text { Object }]_{\varphi}[\text { Verb Complex }]_{\varphi}\right]_{\iota}$

\section{Discussion}

This paper introduces the foundations for Tri-P Mapping, a new model of the phonology-syntax interface. Extreme morpho-syntactic complexity or "polysynthesis" was identified as the crucial test for any interface model, and a side-by-side test using data from Kiowa and Saulteaux Ojibwe showed that no current model accounts for the data in Saulteaux. Relational Mapping correctly predicts the patterns in Kiowa, but it runs into issues as the only model to assume a separate morphological module of grammar. In addition, the intermediate constituent $\kappa$ is imprecisely defined taking away from much of the model's success. Tri-P Mapping achieves the same level of success as Relational Mapping, while also addressing its outstanding issues through referencing prosodic structure to map an intermediate constituent and combining with CoPhonologies by Phase. Three issues remain unresolved and left to future research and discussion: the unexpected phonological boundary and remaining structures in Saulteaux, details of how best to combine Tri-P Mapping and CoPhonologies by Phase, and the precise definition of Constituent $\chi$ and its relationship to compounds.

First, it is necessary to revisit the phonological patterns in Saulteaux that indicate a boundary between inflectional suffixes. Corroborative examples will help illuminate exactly where to place the boundary (e.g. only before the preterit suffix or somewhere else). Of additional interest is the Saulteaux verb stem and is component morphology. The processes examined in Miller 2018 did not indicate other verb-internal domains, 
including preverbal modifiers, but the stem will certainly add to the overall picture of the dialect and language as a whole. Recent work has proposed phonological "zones" within Cree preverbs, a closely related language, and such an analysis may prove insightful in how best to test for further domains in Saulteaux (Kevin Russell, p.c.).

Second, it is clear that combining Tri-P Mapping with CoPhonologies by Phase is advantageous, but it is necessary to examine how the two models may interact at all levels. Morphologically-conditioned phonology is not exclusive to the material between $\omega$ and $\varphi$, so it is worth determining the nature of the combination. It is this and the final questions surrounding $\chi$ that collaborative work underway aims to address (Hannah Sande, p.c.).

Finally, the definition of $\chi$ and its relation to compounds will contribute to the overall discussions surrounding recursion in phonology and how to understand the architecture of phonology as a whole. It is first necessary to revisit and test previous proposals for recursive $\omega$ structures to confirm the necessity of a Constituent $\chi$. As of now, I am unaware of any cases where genuine recursion - as defined above - is present below $\varphi$. If such a case exists, it would make any proposal all the more interesting. In addition, whether or not compounds may be included in a $\chi$ analysis has the potential to alter our understanding of where compounds are situation in the Prosodic Hierarchy. If they are, in fact, separate from $\chi$, should they form a compound-specific constituent? Is that constituent in the same hierarchy? Is it universal? Any answer will push our understanding of phonology and its interface with syntax forward.

\section{References}

Ahn, Byron (2015). Giving Reflexivity a Voice: Twin Reflexives in English. Ph.D. thesis, UCLA.

Bickel, Balthasar \& Johanna Nichols (2001). AUTOTYP database. http://www.autotyp.uzh.ch/.

Bickel, Balthasar, Kristine Hildebrandt \& René Schiering (2009). The distribution of phonological word domains: a probabilistic typology. Phonological Domains: Universals and Deviations, Berlin: Mouton de Gruyter, 47-75.

Bošković, Željko (2016). What is sent to spell-out is phases, not phasal complements. Linguistica 56:1, $25-66$.

Cheng, Lisa \& Laura J Downing (2007). The prosody and syntax of Zulu relative clauses. SOAS Papers in Linguistics 15, 51-63.

Cheng, Lisa Lai-Shen \& Laura J Downing (2016). Phasal syntax= cyclic phonology? Syntax 19:2, 156-191.

Chomsky, Noam (2000). Minimalist inquiries: The framework (mitopl 15). Step by step: Essays on minimalist syntax in honor of Howard Lasnik 89-155.

Chomsky, Noam (2001). Derivation by phase. Kenstowicz, Michael (ed.), Ken Hale: A life in language, Cambridge, MA: MIT Press, 1-52.

Chomsky, Noam (2008). On phases. Current Studies in Linguistics Series 45, p. 133.

Citko, Barbara (2014). Phase theory: An introduction. Cambridge University Press.

Collins, Chris \& Edward Stabler (2016). A formalization of minimalist syntax. Syntax 19:1, 43-78.

Compton, Richard \& Christine Pittman (2007). Affixation by phase: Inuktitut word-formation. The 81st Annual Meeting of the Linguistics Society of America, Anaheim.

Dobashi, Yoshihito (2003). Phonological phrasing and syntactic derivation. Ph.D. thesis, Cornell University.

Dobashi, Yoshihito (2004a). Multiple spell-out, label-free syntax, and PF-interface. Explorations in English Linguistics $19,1-47$.

Dobashi, Yoshihito (2004b). Restructuring of phonological phrases in japanese and syntactic derivation. Manuscript, Kitami Institute of Technology .

Elfner, Emily (2012). Syntax-Prosody Interactions in Irish. Ph.D. thesis, University of Massachusetts - Amherst.

Elordieta, Gorka (2006). Binarity at the left edge of Intonational Phrases. GLOW Workshop on Prosodic Phrasing.

Elordieta, Gorka, Thomas Riad \& Carlos Gussenhoven (2007). A constraint-based analysis of the intonational realization of focus in Northern Bizkaian Basque. T. Riad and C. Gussenhoven, Tones \& tunes 1, 199-232.

Guekguezian, Peter Ara (2017). Prosodic recursion and syntactic cyclicity inside the word. Ph.D. thesis, University of Southern California.

Heinz, Jeffrey \& William Idsardi (2011). Sentence and word complexity. Science 333:6040, 295-297.

Hildebrandt, Kristine A (2007). Prosodic and grammatical domains in Limbu. Himalayan Linguistics 8.

Van der Hulst, Harry (2010). A note on recursion in phonology. Recursion and Human Language 104, p. 301.

Ishihara, Shinichiro (2007). Major phrase, focus intonation, multiple spell-out (MaP, FI, MSO). The Linguistic Review 24:2-3, 137-167.

Jackendoff, Ray \& Steven Pinker (2005). The nature of the language faculty and its implications for evolution of language (reply to Fitch, Hauser, and Chomsky). Cognition 97:2, 211-225.

Kratzer, Angelika \& Elisabeth Selkirk (2007). Phase theory and prosodic spellout: The case of verbs. The Linguistic Review 24:2-3, 93-135.

Ladd, D Robert (1986). Intonational phrasing: the case for recursive prosodic structure. Phonology 3, 311-340. 
Logan, H. J. (2001). A collection of Saulteaux texts with translations and linguistic analyses. Master's thesis, University of Regina.

McPherson, Laura Elizabeth (2014). Replacive grammatical tone in the Dogon languages. Ph.D. thesis, UCLA.

McPherson, Laura \& Jeffrey Heath (2016). Phrasal grammatical tone in the Dogon languages. Natural Language \& Linguistic Theory 34:2, 593-639.

Michaels, Jennifer (2013). To alternate or not to alternate: What is the boundary. Proceedings of 40th meeting of the North East Linguistics Society.

Miller, Taylor (2018). Polysynthesis at the Phonology-Syntax Interface: A Case Study of Kiowa and Saulteaux Ojibwe. Ph.D. thesis, University of Delaware.

Neeleman, Ad \& Jan van de Koot (2006). On syntactic and phonological representations. Lingua 116:10, 1524-1552.

Nespor, Marina \& Irene Vogel (1986). Prosodic Phonology. Walter de Gruyter.

Oxford, Will (2013). Multiple instances of agreement in the clausal spine: Evidence from Algonquian. Proceedings of WCCFL, Citeseer, vol. 31.

Pak, Marjorie (2008). The postsyntactic derivation and its phonological reflexes. Ph.D. thesis, University of Pennsylvania.

Piggott, Glyne \& Heather Newell (2006). Syllabification and the spell-out of phases in Ojibwa words. McGill Working Papers in Linguistics 20:2, p. 39.

Pinker, Steven \& Ray Jackendoff (2005). The faculty of language: what's special about it? Cognition 95:2, 201-236.

Samuels, Bridget D (2009). The structure of phonological theory. Ph.D. thesis.

Samuels, Bridget D (2010). Phonological derivation by phase: Evidence from Basque. University of Pennsylvania Working Papers in Linguistics 16:1, p. 19.

Samuels, Bridget D (2011). Phonological architecture: A biolinguistic perspective, vol. 2. Oxford University Press.

Sande, Hannah (2017). Distributing morphologically conditioned phonology: Three case studies from Guébie. Ph.D. thesis, UC Berkeley.

Sande, Hannah \& Peter Jenks (2017). Cophonologies by phase. North East Linguistic Society (NELS), vol. 48.

Sato, Yosuke (2009). Spelling-out prosodic domains: a multiple spell-out account. Grohmann, K. (ed.), InterPhases: Phase-theoretic Investigations of Linguistic Interfaces, Oxford: Oxford University Press, 234-259.

Sato, Yosuke (2012). Multiple spell-out and contraction at the syntax-phonology interface. Syntax 15:3, $287-314$.

Schiering, René, Kristine A Hildebrandt \& Balthasar Bickel (2007). Cross-linguistic challenges for the prosodic hierarchy: evidence from word domains. Ms. University of Leipzig .

Schiering, René, Balthasar Bickel \& Kristine A Hildebrandt (2010). The Prosodic Word is not universal, but emergent. Journal of Linguistics 46:3, 657-709.

Selkirk, Elisabeth (1980). Prosodic domains in phonology: Sanskrit revisited. Juncture 7, 107-129.

Selkirk, Elisabeth (1981a). On prosodic structure and its relation to syntactic structure. TAPIR Trondheim.

Selkirk, Elisabeth (1981b). On the nature of phonological representation. Advances in Psychology 7, 379-388.

Selkirk, Elisabeth (1995). The prosodic structure of function words. J. Beckman, L. Walsh Dickey \& S. Urbanczyk (eds.), Papers in Optimality Theory, University of Massachusetts Occasional Papers, vol. 18, 439-469.

Selkirk, Elisabeth (2000). The interaction of constraints on prosodic phrasing. Prosody: Theory and Experiment, Springer, 231-261.

Selkirk, Elisabeth (2011). The syntax-phonology interface. John Goldsmith, Jason Riggle \& Alan C. L. Yu (eds.), The Handbook of Phonological Theory, Wiley Online Library, chap. 14, p. 435, 2 edn.

Shwayder, Kobey (2015). Words and subwords: Phonology in a piece-based syntactic morphology. Ph.D. thesis, University of Pennsylvania.

Slavin, Tanya (2005). Evidence for layered morphology: the case of the preverb nihtaa- in Ojibwe. Proceedings of Workshop on Structure and Constituency of Languages of America.

Šurkalović, Dragana (2011). Modularity, linearization and phase-phase faithfulness in Kayardild. Iberia: An International Journal of Theoretical Linguistics 3:1.

Šurkalović, Dragana (2013). Modularity, Phase-Phase Faithfulness and prosodification of function words in English. Nordlyd 40:1, 301-322.

Truckenbrodt, Hubert (1995). Phonological phrases-their relation to syntax, focus, and prominence. Ph.D. thesis, Massachusetts Institute of Technology.

Truckenbrodt, Hubert (1999). On the relation between syntactic phrases and phonological phrases. Linguistic Inquiry $30: 2,219-255$.

Uriagereka, Juan (). Multiple spell-out. Working Minimalism.

Valentine, Randolph J (2001). Nishnaabemwin Reference Grammar. University of Toronto Press.

Vigário, Marina (2010). Prosodic structure between the prosodic word and the phonological phrase: Recursive nodes or an independent domain? The Linguistic Review 27:4, 485-530.

Vogel, Irene (2008). The morphology-phonology interface: Isolating to polysynthetic languages. Acta Linguistica Hungarica 55:1-2, 205-226.

Vogel, Irene (2009a). The status of the clitic group. Phonological domains: Universals and deviations 16, p. 15.

Vogel, Irene (2009b). Universals of prosodic structure. Universals of language today, Springer, 59-82.

Vogel, Irene (2019). Life after the Strict Layer Hypothesis. Zhang, Hongming \& Youyong Qian (eds.), Prosodic Studies: Challenges and Prospects, Routlege.

Voll, R (2006). Prosodic Domains in Richtersveld Nama. Ph.D. thesis, University of Leipzig.

Wagner, Michael (2005). Prosody and recursion. Ph.D. thesis, Massachusetts Institute of Technology.

Watkins, Laurel J. (1984). A Grammar of Kiowa. With assistance of Parker McKenzie. Lincoln: University of Nebraska Press. 